\title{
Analysis of Possible Nonlocal Effects in Laser Beams Generated by Piezoelectric Ceramic
}

\author{
Elio B. Porcelli, Victo S. Filho \\ H4D Scientific Research Laboratory, São Paulo, Brazil \\ Email: elioporcelli@h4dscientific.com
}

How to cite this paper: Porcelli, E.B. and Filho, V.S. (2018) Analysis of Possible Nonlocal Effects in Laser Beams Generated by Piezoelectric Ceramic. Journal of Power and Energy Engineering, 6, 20-32. https://doi.org/10.4236/jpee.2018.62002

Received: January 22, 2018

Accepted: February 6, 2018

Published: February 9, 2018

Copyright $\odot 2018$ by authors and Scientific Research Publishing Inc. This work is licensed under the Creative Commons Attribution International License (CC BY 4.0).

http://creativecommons.org/licenses/by/4.0/

c) (i) Open Access

\begin{abstract}
We report in the present work a novel effect associated with deviations of laser beams when in the surroundings there is a piezoelectric ceramic subjected to high voltages. In our previous work we presented experimental evidences of the generation of forces at distance by piezoelectric materials. Hence a methodology for remote sensing and metrology which enhanced the weak effects of the property of generalized quantum entanglement was proposed. The preexisting state between all existing particles in physical systems can allow that piezoelectric ceramics induct forces at distance detected by a sensible accelerometer apart without involving any known local interaction such as those of acoustic or electromagnetic nature. Motivated by the results, we proceed and discover that new unprecedented visual effects arise when nonlocal forces generated by a piezoelectric ceramic change the laser beam profiles even considering its weakness. This novel effect seems to be really detected in our experiments and this achievement opens one opportunity more for developing new technological applications.
\end{abstract}

\section{Keywords}

Piezoelectric Ceramics, Generalized Quantum Entanglement, Laser Beams, Nonlocal Forces

\section{Introduction}

The piezoelectricity is the phenomenon which appears when one applies in certain materials a mechanical stress and then they generate and maintain electric charges [1] [2] [3]. In the so-called direct effect the piezoelectric materials generate electric charges when stress is applied; in the converse effect, there is the generation of stress when it is applied an external electric field. Under mechanical stress, the materials suffer unbalance in the positive and negative charges in 
their bulk, producing the external electric field described. If the process is reversed, an external electrical field can produce deformations on the piezoelectric material. In present days, such properties of piezoelectric materials have been applied in many technological devices. As examples of relevant applications, we can describe devices for the production and detection of sound, the fabrication of sensors [4], production of filters and other high-frequency applications [5], generation of high voltages and electronic frequency, piezoelectric actuators [6], high voltage actuators in robotic area [7] and ultrasonic motors [8].

We reported in our previous work [9] that piezoelectric materials can generate external induction forces in the environment which are not due to local interactions such as electric field or acoustic wave interactions. As we known, the so-called local interaction means that the interaction is intermediated by some force carriers such as photons that propagate between the interacting parts of the physical system.

Our experimental and theoretical investigation points out that attractive or repulsive nonlocal induction forces can be performed when piezoelectric materials suffer respectively a mechanical contraction or stretching. The direction and the signal of the force induced depend on the direction of the physical mechanical change. The intensity of the nonlocal force generated depends on either the magnitude of the voltage applied and material characteristics in case of converse mode or the mechanical stress applied and also material characteristics in case of direct mode. The magnitude and direction of the nonlocal force can be measured by accelerometers accordingly.

Considering all those developed concepts, it was applied a patent [10] related to the new methodology for metrology and remote sensing in which the piezoelectric devices are "emitters" of nonlocal forces in some special conditions and accelerometers are "detectors".

The nonlocal forces occur between quantum entangled particles of the system where their momentum variations can be exchanged directly with no dependence on the medium and the force carriers.

We also indicated that there is a preexisting condition of generalized quantum entanglement (GQE) between all existing particles [11] which is usually extremely weak in terms of its effects. However it can be amplified in some special conditions existing in piezoelectric materials operating in the direct or reverse mode.

The results of this research encouraged us to analyze if the nonlocal force induction generated by piezoelectric materials could be strong enough to affect the laser beams considering that just sensible accelerometers were implemented to detect it so far.

In next section, we present details of our experiments, involving the PZT4 piezoelectric ceramic and laser beams generated by usual laser pointers. In the following, we present an analysis of the video frames captured during the experiments outstanding the novel visual effect detected. Next, we present a theoretical 
analysis of the novel visual effect and the difficulties for explain it via usual theoretical framework. Afterwards we present an analysis of the novel visual effect and its qualitative explanation according to the GQE framework. As last we discuss our main conclusions.

\section{Description of the Experiments}

\subsection{Introduction}

The experimental setup consists of a laser pointer supported by a tripod and a 5 $\mathrm{cm}$ diameter and $2.5 \mathrm{~mm}$ thickness PZT4 ceramic disc supported with its circular face in a horizontal position on a light Styrofoam holder. The mentioned disc is also positioned below and immediately near the laser beam output of the pointer. When triggered, the laser beam passes a few millimeters above the circular face of the disc crossing above its center and also its entire diameter.

The circular faces of the disc are covered by metallic finish and they are connected to a high voltage power supply via aluminum stripes. When triggered, the laser beam is projected over a certain distance and achieves almost perpendicularly a whiteboard with 1 -centimeter grid. The grids are used as the position reference for analysis of the shape change of the laser beam profile projection.

In some outdoor or external tests, the power cord of the high voltage power supply was plugged in the inverter connected to +12 VDC output of the car in order to supply $110 \mathrm{VAC}$. In other indoor tests, the power cord was plugged directly in the AC outlet of the laboratory.

Three different distances were tested such as $10.85 \mathrm{~m}, 47 \mathrm{~m}$ and $120 \mathrm{~m}$ between the whiteboard and laser pointer in order to project some laser beam profiles with respectively $2 \mathrm{~cm}, 5 \mathrm{~cm}$ and $9 \mathrm{~cm}$ in terms of average diameter. In the first and last distances, the experimental setup was performed in the field (outdoor) with cloudy sky, more than $85 \%$ air relative humidity and around $21^{\circ} \mathrm{C}$ temperature in terms of weather conditions. In the second distance, the setup was performed inside the building (indoor) with the PZT4 ceramic disc covered by two thin layers of polystyrene in order to even more reduce at least 2.3 times the electric field around it.

The laser pointer had a continuous activation in order to project the laser beam. During its activation, it was applied a DC high voltage on the PZT4 ceramic disc with approximately $1 \mathrm{kV}$ by the power supply during approximately 30 seconds per each cycle. In other words, we had approximately 15 seconds "off" with $0 \mathrm{~V}$ and 15 seconds "on" with up to $1 \mathrm{kV}$ per cycle in terms of alternating operation.

The PZT4 ceramic disc was tested in the converse piezoelectric mode, that is, when a mechanical stress is generated according to the voltage applied on it. The disc was polarized directly with the positive pole of the power supply connected to the positive side of the disc and the negative pole of the power supply connected to the negative side of the disc. In other tests, the disc was reversely polarized, that is, the positive pole of the power supply was connected in the negative 
side of the disc and the negative pole of the power supply was connected in the positive side. When the PZT4 ceramic disc is polarized directly, its thickness has a mechanical stretching in the $\mathrm{z}$ axis and it has a mechanical contraction in the same direction when polarized reversely.

During the tests, several videos of the laser beam profile projected to the whiteboard were performed and several frames of them were captured accordingly.

The list of devices performed in the experiments is shown in Table 1.

The Figure 1 shows the photo of the experimental setup taken inside the

Table 1. Devices used in the experimental setup conceived in order to measure possible effect of the piezoelectric ceramic on laser beams.

\begin{tabular}{|c|c|}
\hline Device & Features/Specifications \\
\hline PZT4 Disc & $\begin{array}{c}\text { Device provided by ATCP do Brasil, } 5 \mathrm{~cm} \text { diameter, } \\
2.5 \mathrm{~mm} \text { thickness, } 913.2 \mathrm{KHz} \text { resonance frequency, } 8.86 \mathrm{nF} \\
\text { capacitance, } 1419.64 \text { epsilon relative }\end{array}$ \\
\hline $\begin{array}{c}\text { Non Brand } \\
\text { High-Voltage Power Supply }\end{array}$ & $\begin{array}{c}\text { Model AT } 30 \mathrm{KV} \text {, output range } 0 \text { - } 27 \mathrm{kV} \mathrm{DC} \text {, voltage resolution } \\
100 \mathrm{~V} \text { Ripple } 150 \mathrm{~V} \text {, Standard output current } 0.3 \mathrm{~mA} @ 15 \mathrm{kV} \text {, } \\
\text { Power } 20 \mathrm{~W} \text {, Input AC voltage } 127 \text { or } 220 \mathrm{VAC}\end{array}$ \\
\hline Laser Pointer & $\begin{array}{c}\text { Laser with } 100 \mathrm{~mW} \text { maximum output power, } 523 \mathrm{~nm} \text { wavelength } \pm 10 \text {, } \\
\text { class III laser diode, green color }\end{array}$ \\
\hline Car Inverter Device & $\begin{array}{l}12 \mathrm{VDC} \text { input, } 110 \mathrm{VAC} \text { output, } 150 \mathrm{~W} \\
\text { power rating, cigarette light plug }\end{array}$ \\
\hline Whiteboard & 1-centimeter grid, manufactured by Science First \\
\hline Apple Smartphone & $\begin{array}{l}\text { Model iPhone X, } 12 \mathrm{MP}, f / 1.8 \text { aperture of wide-angle, } f / 2.4 \\
\text { aperture of telephoto, digital zoom up to } 10 \mathrm{x} \text { of optical zoom, } 4 \mathrm{~K} \\
\text { video recording at } 24 \mathrm{fps}, 30 \mathrm{fps} \text { or } 60 \mathrm{fps}\end{array}$ \\
\hline
\end{tabular}

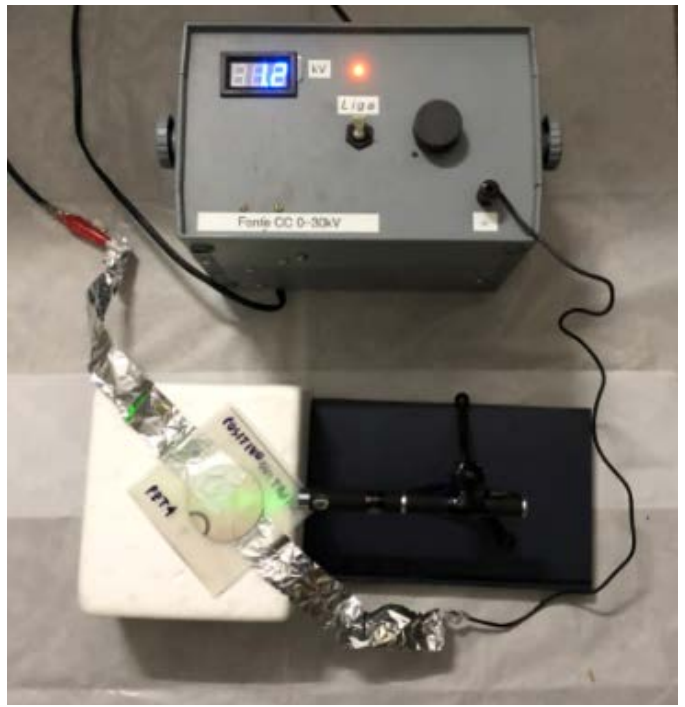

Figure 1. The experimental setup performed inside the building (indoor) with $47 \mathrm{~m}$ laser beam length. It is shown the high voltage power supply (grey box) where the PZT4 ceramic disc covered by thin layers of acetate is connected by strips of aluminum. The disc is supported by the white styrofoam box. The laser pointer supported by a tripod has continuous activation. 
laboratory building, with a $47 \mathrm{~m}$ in terms of distance between output of laser beam pointer and white board.

The Figure 2 and Figure 3 show the photos of the experimental tests performed in the field (outdoor).

\subsection{Analysis of the Video Frames Recorded in the Experiments}

It was made a comparison between the video frames obtained in the "on" period (high voltage applied in the PZT4 ceramic disc) and "off" period (power supply turned off) and it was noted a visual subtle difference mainly in some regions of

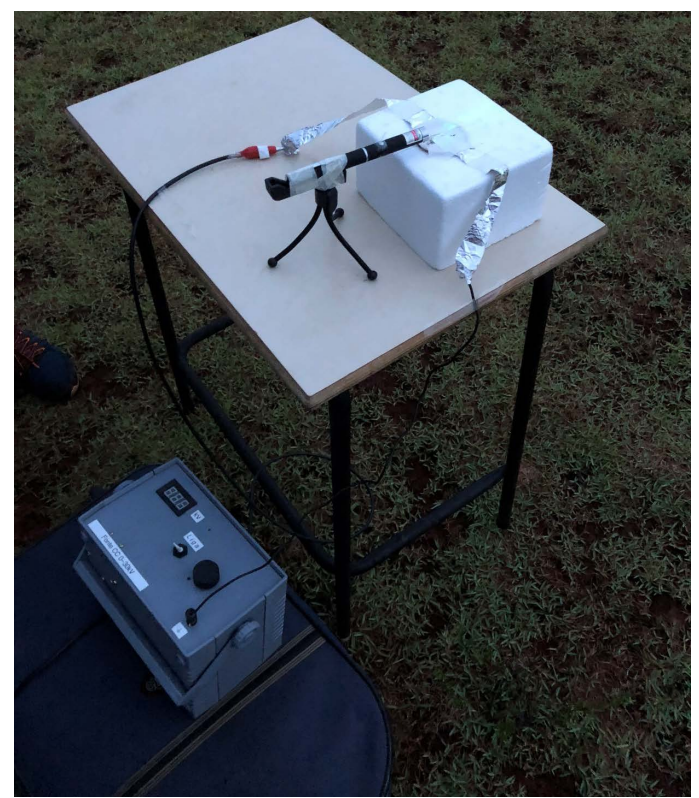

Figure 2. The experimental setup performed in the field (outdoor) with $120 \mathrm{~m}$ laser beam length. It is shown the high voltage power supply (grey box) where the PZT4 ceramic disc supported on the styrofoam box is connected by strips of aluminum. The laser pointer is supported by a tripod and it is activated continuously.

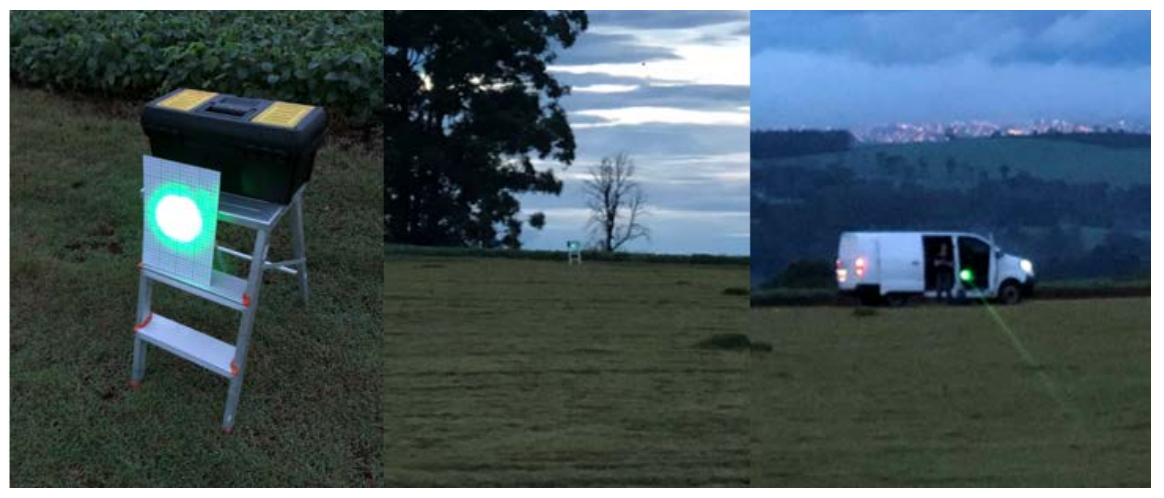

Figure 3. The three photos show different views of the experimental setup used in the field (outdoor). The left photo shows the laser beam profile projected in the whiteboard with 1-centimeter grid. The middle photo shows the mentioned devices that are far $120 \mathrm{~m}$ from the laser pointer. The right photo shows the position of the laser pointer far $120 \mathrm{~m}$ from the whiteboard. 
the laser beam profile projected in the whiteboard as its upper, bottom, left and right corners.

It was also just considered the analysis of the video frames after several seconds from the short period of power supply activation in order to the high voltage be well stabilized and to avoid any possible transitory acoustic propagation in the air during the short period of stretching or contraction of the PZT4 ceramic disc.

Some small variation (blinking, oscillation) of the laser beam profile did not strongly affect the visualization of the differences between the video frames from the "on" and "off" periods.

Considering all of the tests, the shape of the laser beam profile seems to be stretched in the upright direction ( $\mathrm{z}$ axis) and contracted at the same time in the horizontal direction during the "on" period when the PZT4 ceramic disc was directly polarized, that is, when its thickness was stretched.

It was noted the opposite, that is, the laser beam profile seems to be contracted in the upright direction ( $\mathrm{z}$ axis) and stretched at the same time in the horizontal direction during the "on" period when the PZT4 ceramic disc was inversely polarized, that is, when its thickness was contracted.

The laser beam profile recovered its original symmetric shape when it was again turned off the voltage (off period).

The possible scenarios are represented in three diagrams drawn at the top of the Figure 4, where we can see from the left to the right, the original symmetric condition of the laser beam profile shape, the condition of contraction in the $\mathrm{z}$ axis when the disc is reversely polarized and the condition of stretch in the $\mathrm{z}$ axis when the disc is directly polarized. For didactic proposal, the shape variation drawn in the diagrams was exaggerated. The simple diagram of the experimental setup including the laser pointer, laser beam projection, PZT4 ceramic disc, the whiteboard and the laser beam profile is drawn in the bottom of the Figure 4.

The dimension of the shape of the laser beam profile seems to change approximately $0.5 \mathrm{~mm}$ in case of the test for a $10.85 \mathrm{~m}$ distance.

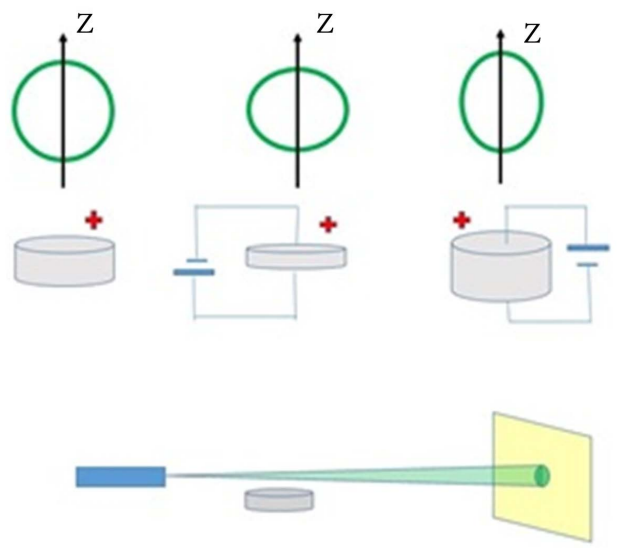

Figure 4. The conditions of the laser beam profile are drawn in three diagrams at the top. The simple diagram of the experimental setup is drawn in the bottom. 
Considering that the PZT4 ceramic disc affected the laser beam emitted above it during the short "on" time, it is reasonable to obtain probably higher dimension shape changes for long distances. Therefore, it was performed other tests afterwards for long distances, such as $47 \mathrm{~m}$ and $120 \mathrm{~m}$, which resulted significant dimension shape changes, that is, respectively $2 \mathrm{~mm}$ and $5 \mathrm{~mm}$, with $20 \%$ estimated error.

The Figure 5 shows four video frames obtained in the experiments performed with 120 m distance where we can see two laser beam profiles "off" and "on" in case of PZT4 ceramic disc polarized reversely (contraction) and also two profiles "off" and "on" in case of ceramic disc polarized directly. The subtle contraction of the laser beam profile in the $\mathrm{z}$ axis is visible for the reverse polarization of the disc and the subtle stretching of the laser beam profile in the $\mathrm{z}$ axis is visible for the direct polarization.

Such as in Figure 5, Figure 6 also shows four video frames obtained in the experiments performed with $47 \mathrm{~m}$ distance where we can see two laser beam profiles "off" and "on" in case of PZT4 ceramic disc reversely polarized (contraction). Analogously, we also have two profiles "off" and "on" in case of ceramic disc directly polarized. As expected from the earlier experimental result, the subtle contraction of the laser beam profile in the $\mathrm{z}$ axis is visible for the reverse polarization of the disc and the subtle stretching of the laser beam profile in the $\mathrm{z}$ axis is visible for the direct polarization.

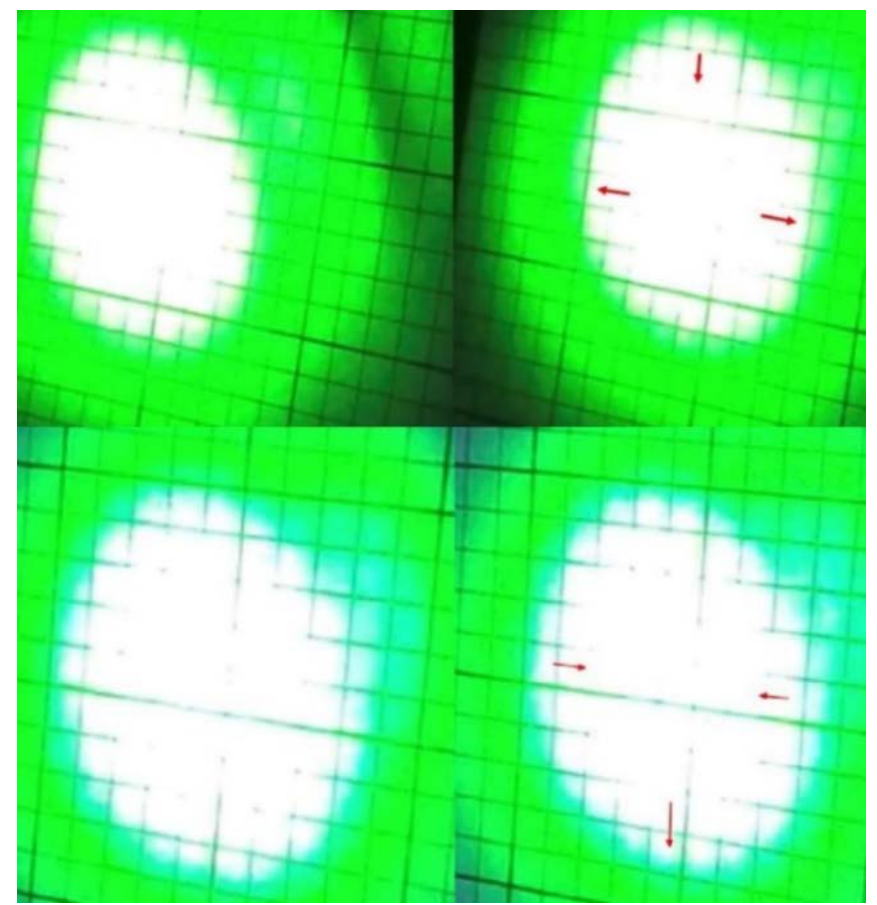

Figure 5. Some of several video frames of laser beam profile captured during the video with $120 \mathrm{~m}$ laser length. We can see the frames of the periods as reverse "off", reverse "on", direct "off", direct "on" respectively in top left, top right, bottom left and bottom right. The arrows show the direction of the small displacement of the laser beam profile (5 mm maximum observed). 


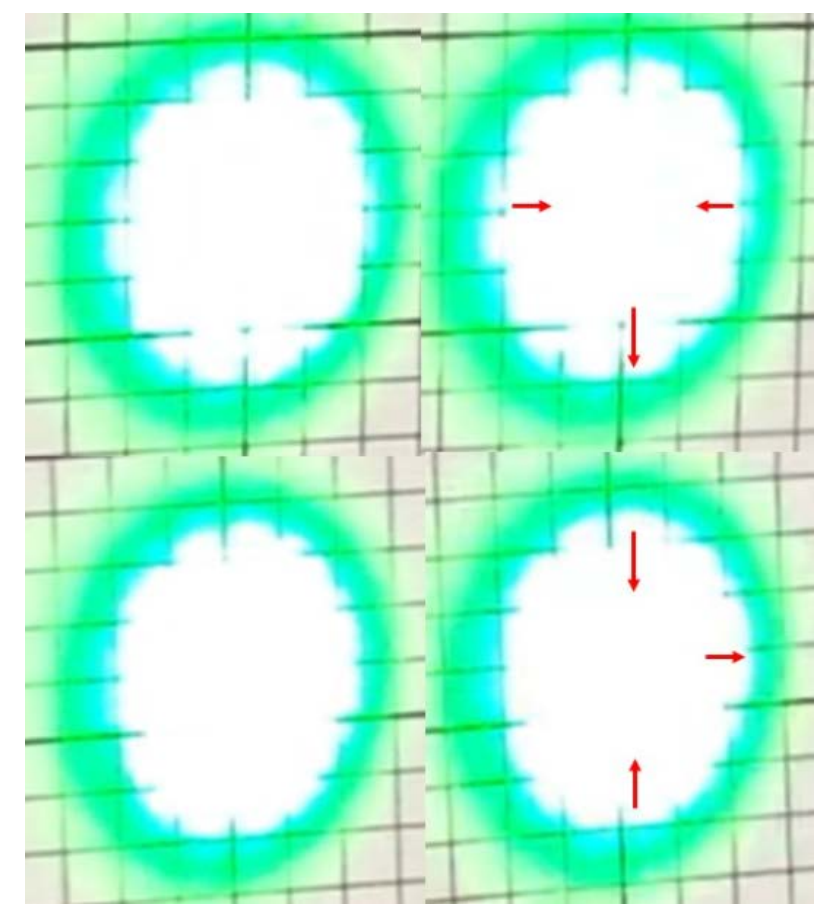

Figure 6. Some of several video frames of laser beam profile captured during the video with $47 \mathrm{~m}$ laser length. We can see the frames of the periods as reverse "off", reverse "on", direct "off", direct "on" respectively in top left, top right, bottom left and bottom right. The arrows show the direction of the small displacement of the laser beam profile $(2 \mathrm{~mm}$ maximum observed).

\section{Theoretical Explanation}

\subsection{Description of the Novel Effects via Usual Theoretical Framework}

There is no acoustic interaction from the PZT4 disc in its converse mode to the laser beam because it was applied a DC voltage (static electric field) and the visual analysis of the laser beam profiles just consider the video frames after the short time (milliseconds) of the high voltage activation.

Since the low power laser beam is not affected by traveling through static electric field [12], therefore this cannot explain the change of the profiles shape projection that we analyzed.

Thinking about some other conventional explaining, there is the electro-optical Kerr effect in the atmosphere [13] where a static electric field causes an alignment of the air molecules, and creates birefringence, that is, the optical path length change $(\Delta n)$ can happen according to the following equation:

$$
\Delta n=\lambda K \varepsilon^{2},
$$

in which $\lambda$ is the vacuum wavelength of the laser beam, $K$ is the Kerr constant of the air (almost $2.3 \times 10^{-25} \mathrm{~m}^{2} \cdot \mathrm{V}^{-2}$ ) and $\varepsilon$ is the magnitude of the static electric field applied.

It is well noted in the Equation (1) that the optical path length change $(\Delta n)$ varies according to the square value of the electric field. In our case it is $0.4 \times 10^{6}$ 
$\mathrm{V} / \mathrm{m}$ (1 kV DC voltage applied) between the circular surfaces of the PZT4 disc separated by a $2.5 \mathrm{~mm}$ thickness.

The electro-optical Kerr effect occurs in some condition in which a static electric field is applied in the direction transverse to the laser beam direction and this condition can theoretically happen in our experiments. After all, the static electric field applied between the circular faces of the PZT4 disc is perpendicular to the laser beam positioned a few millimeters above it.

Some static electric field can emerge from the top of the circular face of the PZT4 disc causing the alignment of the air molecules placed on it where the laser beam propagates. Despite of this, according to [13], regarding the electro-optical Kerr effect in the atmosphere, for most cases, the laser beam phase shift and its variation are so small as to render its proposed measurements unfeasible. The reference [13] assumed a resolution of $2 \times 10^{-8}$ for measured valued of phase shift.

The magnitude of the electric field on the top of the PZT4 disc axis must be reduce around $92 \%$ in the laser beam position of propagation (just 2 millimeters above), that is, $0.368 \times 10^{6} \mathrm{~V} / \mathrm{m}$ for a $1 \mathrm{kV}$ DC voltage applied. Remind that the low power laser beam isn't affected in its propagation through a static electric field [12]. Considering this value of electric field (the electric field magnitude also decays from the center of the disc towards the edge, following the radius direction) and the short path length of the laser beam (PZT4 disc diameter) of $0.05 \mathrm{~m}$, the overestimated phase shift value is calculated as $1.963 \times 10^{-8}$ according to Equation (1). Therefore this value is less than the mentioned resolution limit of measurement $\left(2 \times 10^{-8}\right)$.

It was also applied some dielectric layers of thin polystyrene foils (dielectric constant equal to 2.3) covering the PZT4 disc in case of experiments performed indoor in order to reduce even more some possible emerging electric field remained in the environment. In this condition, the overestimated phase shift is calculated as $0.371 \times 10^{-8}$, that is, much less than the resolution limit of measurement. We need to outstand that the changes in the laser beam profiles shape with the $1 \mathrm{kV}$ DC voltage application in the PZT4 disc remained the same with or without the dielectric layers coverage.

All these arguments strongly suggest that it is not easy to explain the novel visual effects via some conventional theoretical framework, so that local interactions such as acoustic or electromagnetic ones cannot probably be responsible for them.

\subsection{Qualitative Explanation of the Novel Effects via GQE Approach}

In our previous works, novel effects detected in experiments performed by ourselves and other authors such as in symmetric capacitors [14] [15] asymmetric capacitors [16], magnetic cores [11], laser diodes [17], piezoelectric devices [9] [10] and superconductor devices [18] [19] seem to be quantitatively and qualitatively explained by an unique theoretical framework. In this framework [11], the preex- 
isting condition of generalized quantum entanglement (GQE) between all existing particles in a physical system, usually extremely weak because of the huge entropy, rises when a myriad of group of particles are conditioned to have the same quantum state (local entropy reduction) via a strong local field such as electric or magnetic one, for example. In the present work we report that the anomalous forces verified can be microscopically originated from the state of generalized quantum entanglement of their particles, in a manner similar to the anomalous effects analyzed in other physical systems mainly based on dipoles, dielectric materials and superconductors. The difference between the anomalous effect raised from piezoelectric materials and each previous physical system analyzed is in the type of the source device and the nature of the effect. For instance, in case of capacitors under high voltage, it appears on them an upward anomalous force decreasing their apparent weight and in case of superconductors and diode laser beams it is produced a field of forces at distance in the surroundings.

There is also a favorable condition for GQE effects rising in case of the molecular electric dipoles inside the piezoelectric ceramic disc (over high voltage) inducting its dipolar momentum variations to the group of the laser beam photons positioned at distance in the experiments described in this work.

The data information of PZT4 ceramic disc (according to our previous article [9]) allows us to calculate the intensity of the attractive or repulsive on local force inducted to the laser beam. The modulus of the force for the $1000 \mathrm{~V}$ voltage applied in the piezo electric ceramic disc can be calculated using $F=V \cdot T / g_{33}$ as follows:

$$
F=V \cdot T / g_{33}=1000 \times 2.5 \times 10^{-3} / 0.02292=109.1 \mathrm{~N} .
$$

The second step is to calculate the strain $S$, using:

$$
S=F / A=109.1 /\left(1.964 \times 10^{-3}\right)=55564.17 \mathrm{~N} / \mathrm{m}^{2},
$$

in which $A$ is the circular area of the disc. The next step is to calculate the physical deformation $D$ using the equation

$$
D=S / Y_{33}=55564.17 /\left(6.2 \times 10^{10}\right)=8.962 \times 10^{-7} .
$$

Finally, then on local force induction can be calculated as shown:

$$
f=F \cdot D=109.1 \times 8.962 \times 10^{-7}=9.78 \times 10^{-5} \mathrm{~N} .
$$

In our next work, this value of non local force will be used for predicting the displacement of the laser beam profile in term sofa quantitative analysis.

When the PZT4 ceramic disc in piezoelectric converse mode condition is directly polarized via DC voltage application, the internal (lead zirconate titanate) electric molecular dipoles change its collective dipolar momentum in order to stretch the thickness dimension of the disc both upwards and downwards ( $\mathrm{z}$ axis). Theoretically, the group of photons of the laser beam crossing above the disc projection area also presents a change in its transversal momentum component ( $\mathrm{z}$ axis) because of the nonlocal induction generated by the molecular dipoles of the disc. The stretching of the laser beam profile projected in the white- 
board is visually observed accordingly.

In the opposite case, that is, when the PZT4 ceramic disc in piezoelectric converse mode condition is inversely polarized via DC voltage application, the electric molecular dipoles change its collective dipolar momentum in order to contract the thickness dimension of the disc. The group of photons of the laser beam has also change in its transversal momentum component ( $\mathrm{z}$ axis) because of the nonlocal induction of molecular dipoles of the disc and the contraction of the laser beam profile projected in the whiteboard is also visually observed accordingly.

In case of the non-energized condition of the PZT4 ceramic disc, that is, when we have no voltage applied, the internal electric molecular dipoles are not subjected to any electric field and it cannot change the transversal momentum component of the group of laser beam photons. In this condition, there is no visual change in the laser beam profile and it can remain with its original symmetric shape.

In terms of quantum framework, the system electric dipole-photon presented in our experiments has some similarity with other experiment [20] where a single photon interacted with a single atom (ion, electric dipole) and its state was measured to be analyzed afterwards. In other similar experiment [21] we had the opposite, a single atom (ion, electric dipole) interacted with a single photon and its state was measured to be also analyzed. In both experiment, the coherence of the quantum states of both the photon and the atom (ion, electric dipole) was well controlled for the detection of quantum entanglement between both where the state variation of one of the two entailed the variation of the state of the other.

In our experiments, it is supposed that a myriad of internal electric dipoles of the PZT4 ceramic disc submitted to the same local electric field can change its quantum state and also the quantum state of a myriad of coherent photons emitted from the laser pointer considering the preexisting condition of generalized quantum entanglement (GQE) between them. The change in the macroscopic observable, that is, in the shape dimensions of the laser beam profile follows the change in the other macroscopic observable (thickness of PZT4 ceramic disc).

For the next step, the authors intend to perform more experiments in order to better characterize the magnitude of the changes of the laser profile shape according to the magnitude of the induction generated by the PZT4 ceramic disc already characterized by accelerometer measurements. More experiments related to the measure of the geometry of the induction will be also performed.

\section{Conclusion}

In this work, we present a novel effect characterized by the change in the shape of a laser beam profile when the PZT4 ceramic disc in the piezoelectric converse mode is directly or reversely polarized via DC high voltage applied. The shape of the laser beam profile follows the change of the thickness dimension of the disc, so that it can contract or stretch in the vertical or horizontal direction. The en- 
hancement of the novel visual observation of this subtle effect was achieved for higher distances between the laser pointer and whiteboard. We present all experimental details and the main difficulties to explain the effect via usual theoretical framework. We also propose the explanation of the effect via GQE framework such as performed in our other previous works. The novel visual effect seems to be explained qualitatively in accordance with the GQE framework. According to this framework, the change of the collective quantum state of the internal molecular dipoles of the ceramic disc over the electric field inducts the change in the collective quantum state of the laser beam photons considering their preexisting mutual photon-dipole state of quantum entanglement. In our earlier work, the weak induction of the so considered nonlocal forces generated by piezoelectric materials was only detected via accelerometer, but this novel visual effect makes the phenomenon even more perceptible and opens new possible technology applications. It is important to reinforce that our experimental setup represents an original concept and we do not have other experimental works verifying such a property in order to compare the results. However, we intend to analyze such a novel effect more profoundly in future works. The authors also intend to keep researching this new effect in order to characterize and theoretically quantify it accordingly.

\section{References}

[1] Cady, W.G. (1964) Piezoelectricity. McGraw-Hill, New York.

[2] Jaffe, B., Cook, W. and Jaffe, H. (1971) Piezoelectric Ceramics. Academic Press, London.

[3] Holterman, J. and Groen, P. (2013) An Introduction to Piezoelectric Materials and Applications. Stichting Applied Piezo, Apeldoorn.

[4] Gautschi, G. (2002) Piezoelectric Sensorics. Springer Berlin Heidelberg, Berlin.

[5] Newnham, R.E., Bowen, L.J., Klicker, K.A. and Cross, L.E. (1980) Composite Piezoelectric Transducers. Materials \& Design, 2, 93-106.

https://doi.org/10.1016/0261-3069(80)90019-9

[6] Uchino, K. (1986) Piezoelectric/Electrostrictive Actuators. Morikita Publishing, Tokyo.

[7] Karpelson, M., Wei, G.-Y., and Wood, R.J. (2012) Driving High Voltage Piezoelectric Actuators in Microrobotic Applications. Sensors and Actuators A: Physical, 176, 78-89. https://doi.org/10.1016/j.sna.2011.11.035

[8] Uchino, K. (1997) Piezoelectric Actuators and Ultrasonic Motors. Kluwer Academic Publishers, Boston.

[9] Porcelli, E.B. and Filho, V.S. (2018) Induction of Force Performed by Piezoelectric Materials. JPEE, 6, 33-50.

[10] Porcelli, E.B. (2015) Induction of Force Performed by the Piezoelectric Materials. US Patent No. 2015/0188026 A1.

[11] Porcelli, E.B. and Filho, V.S. (2017) Anomalous Effects from Dipole-Environment Quantum Entanglement. International Journal of Engineering Science, 4, 131-144. https://doi.org/10.22161/ijaers.4.1.21

[12] Bulanov, S., Esirkepov, T., Thomas, A., Koba, J. and Bulanov, S. (2010) Schwinger 
Limite Attainability with Extreme Power Lasers. Physical Review Letters, 105, 220407-220410. https://doi.org/10.1103/PhysRevLett.105.220407

[13] Weinheimer, A.J. (1985) The Electro-Optical Kerr Effect in the Atmosphere. JTECH, 3, 175-178.

[14] Porcelli, E.B. and Filho, V.S. (2015) On the Anomalous Weight Losses in High-Voltage Symmetrical Capacitors.

[15] Porcelli, E.B. and Filho, V.S. (2016) On the Anomalous Forces in High-Voltage Symmetrical Capacitors. Physics Essays, 29, 2-9. https://doi.org/10.4006/0836-1398-29.1.002

[16] Porcelli, E.B. and Filho, V.S. (2016) Characterisation of Anomalous Asymmetric High-Voltage Capacitors. IET Science, Measurement \& Technology, 10, 383-388. https://doi.org/10.1049/iet-smt.2015.0250

[17] Porcelli, E.B. and Filho, V.S. (2017) Induction of Forces at Distance Performed by Semiconductor Laser Diodes. American Journal of Engineering Research, 6, 35-48.

[18] Porcelli, E.B. and Filho, V.S. (2017) Theoretical Study of Anomalous Forces Externally Induced by Superconductors. Journal of Natural Sciences, 9, 293-305.

[19] Porcelli, E.B. and Filho, V.S. (2018) Analysis of Possible Nonlocal Forces in Superconducting Materials.

[20] Monroe, D., Meekhof, M., King, B.E. and Wineland, D.J. (1996) A "Schrödinger Cat" Superposition State of an Atom. Science, 272, 1131-1136. https://doi.org/10.1126/science.272.5265.1131

[21] Brune, M., Hagley, E., Dreyer, J., Maitre, X., Maali, A., Wuonderlich, C., Raimond, J.M. and Haroche, S. (1996) Observing the Progressive Decoherence of the "Meter" in a Quantum Measurement. Physical Review Letters, 77, 4887-4890.

https://doi.org/10.1103/PhysRevLett.77.4887 